\title{
Nonlinear effects in the regularity problems for infinite dimensional evolutions of unbounded spin systems
}

\author{
Alexander Val. Antoniouk*, Alexandra Vict. Antoniouk \\ Department of Nonlinear Analysis, \\ Institute of Mathematics NAS Ukraine, \\ Tereschenkivska 3, 01601 MSP, Kiev 4, Ukraine
}

Received August 15, 2005, in final form January 31, 2006

\begin{abstract}
We study the regularity problems for unbounded spin systems of anharmonic oscillators, that approximate multi-dimensional Euclidean field theories. The main attention is paid to the effect of anharmonism on the $C^{\infty}$-regularity properties of evolutional semigroup. Our approach is based on a new class of nonlinear estimates on variations, that permit to obtain regular properties for essentially nonlinear equations.
\end{abstract}

Key words: anharmonic lattice spin systems, nonlinear regularity problems

PACS: $02.30 . J r, 02.30 . S a, 02.30 . X x, 02.50 . E u, 03.654 . D b, 05.50 .+q, 75.10 . H k$

\section{Introduction}

Presently, a rigorous study of numerous important physical models turns out to be impossible without elaboration of mathematical tools inherent to these models and without solution of pure mathematical problems that require the development of adequate calculus.

Many physical processes may be described in terms of infinite dimensional stochastic differential equations of general form

$$
\mathrm{d} \xi^{x}(t)=-G\left(\xi^{x}(t)\right) \mathrm{d} t+D\left(\xi^{x}(t)\right) \mathrm{d} W(t), \quad \xi^{x}(0)=x,
$$

where $W$ is a Brownian motion representing some heat source, $D$ is the diffusion coefficient of inhomogeneous medium and the drift part $G$ corresponds to the force existing in the system, which keeps the system at equilibrium. The corresponding semigroup $\left(P_{t} f\right)(x)=\mathbf{E} f\left(\xi^{x}(t)\right)$, constructed as a mean $\mathbf{E}$ with respect to the Wiener measure, describes the heat evolution of a corresponding physical medium.

Traditionally the linear part $B$ of force $G\left(\xi^{x}(t)\right)=\left[B \xi^{x}(t)+F\left(\xi^{x}(t)\right)\right]$ is separated from nonlinear term $F$. Since in many applications $B$ is given by some unbounded operator, the research mainly concentrated on the case of unbounded $B$, which became a topic of the stochastic partial differential equations theory, e.g. $[1,2]$. At the same time, the simplified classes of nonlinear $F$ of the most linear order of growth, the so-called globally Lipschitz nonlinearities (i.e. when $\| F(x)-$ $F(y) \| \leqslant$ const $\|x-y\|)$, were considered. The case of non-Lipschitz coefficients was successfully treated only for partial problems, such as existence, uniqueness and ergodic behaviour of solutions, e.g. [1-4] and references therein.

However, many important problems of modern physics contain essential nonlinearities that cannot be handled with the tools developed for the models with Lipschitz nonlinearities. The most striking example is the diverging perturbation series for Euclidean fields with nonlinear interactions in high dimensions. Similar complications arise in other important physical models, e.g. [5,6].

In this article we discuss regularity properties of evolutions with non-Lipschitz nonlinearities, i.e. we study how nonlinearity of the problem (1.1) effects the regular dependence of the process $\xi^{x}(t)$ with respect to initial data $x$ and what requirements the topologies of spaces of differentiable

\footnotetext{
*E-mail: antoniouk@imath.kiev.ua
} 
functions should meet to permit the construction of semigroup $P_{t}$ in these spaces. Though the monotonicity conditions of coefficients of (1.1), which lead to the existence and uniqueness of nonlinear process $\xi^{x}(t)$ and its semigroup $P_{t}$, are known long ago $[3,4]$ and the question of regularity has already been raised in literature, one may consult e.g. $[1,2,7]$ and the most recent $[8,9]$ to see that the final solution is still far from reach.

A question arises here. Which methods from nonlinear analysis or stochastic theory can be applied to the investigation of regularity properties in non-Lipschitz case? The application of the classical tools of nonlinear analysis, such as implicit function theoremes, finite dimensional Galerkin or Yosida approximations of nonlinearity $F$ (in order to get the regularity properties of $\xi_{t}^{x}$ and $P_{t}$ from the regularity properties of approximating problems) would be complicated. First of all, the nonlinear mappings in infinite dimensional space are mostly non-Lipschitz even locally on balls. Moreover, as it is discussed in [10] and [11, §1.1], the use of standard topologies of spaces of continuously differentiable functions for regularity problems is not viable in non-Lipschitz case. Each kind of nonlinearity requires guessing certain corrections of topologies of the classical functional spaces and such corrections are not visible at the level of approximations.

One may also attempt the tools of stochastic theory, such as Girsanov transformation, BismutElworthy formula and the related approach of Malliavin calculus, e.g. [12,13]. Though Girsanov transformation removes the nonlinear drift from equation (1.1), the developed techniques will not be adapted to the general diffusion coefficient $D$ in (1.1). The applications of Bismut-Elworthy formula are possible, e.g $[2,11,14-17]$, but these methods are more adapted to the study of differentiability of the process $\xi^{x}(t)$ with respect to the random parameter and actually require a bit of work in nonLipschitz case. So, their development would be more oriented to the mastering of stochastics and would be indirect to the pure nonlinear analytical problem about the regularity of non-Lipschitz equations (1.1) and their semigroups.

The question is whether the direct work with regularity properties for nonlinear equations (1.1) is possible at all? To find an answer we should ask what is actually nonlinearity. One may say that it is a nonlinear response to linear operations. For example, for the linear differentiation operation $\partial_{x}$ one has by chain rule

$$
\partial_{x}^{(n)}(f \circ g)(x)=\sum_{j=1}^{n} \sum_{k_{1}+\cdots+k_{j}=n}\left(f^{(j)} \circ g\right)(x) \partial_{x}^{\left(k_{1}\right)} g(x) \cdots \partial_{x}^{\left(k_{j}\right)} g(x) .
$$

The consideration of terms with $j=1$ and $j=n$ displays symmetry

$$
\partial_{x}^{(n)} g(x) \sim\left[\partial_{x} g(x)\right]^{n}
$$

that holds for all $n \in \mathbb{N}$ and is present in all intermediate terms due to

$$
\partial_{x}^{\left(k_{1}\right)} g(x) \ldots \partial_{x}^{\left(k_{j}\right)} g(x) \sim\left[\partial_{x} g(x)\right]^{k_{1}} \ldots\left[\partial_{x} g(x)\right]^{k_{j}} \sim\left[\partial_{x} g(x)\right]^{k_{1}+\cdots+k_{j}} \sim\left[\partial_{x} g(x)\right]^{n} .
$$

In this article we discuss the consequences of symmetry (1.2) for nonlinear equations (1.1). We develop the results of $[10,16,17]$ and consider an infinite dimensional model of interacting particles with unbounded spins, that, in particular, approximates the high dimensional Euclidean fields with nonlinear interaction $[5,6]$.

\section{Description of model and nonlinear estimate on variations}

Consider a particular case of stochastic differential equation (1.1):

$$
\left\{\begin{array}{l}
\mathrm{d} \xi_{k}^{x}(t)=\mathrm{d} W_{k}(t)-\left\{F\left(\xi_{k}^{x}(t)\right)+\left(B \xi^{x}(t)\right)_{k}\right\} \mathrm{d} t \\
\xi_{k}^{x}(0)=x_{k}, \quad k \in \mathbb{Z}^{d}
\end{array}\right.
$$

Here $k=\left(k_{1}, \ldots, k_{d}\right)$ is a point of $d$-dimensional lattice $\mathbb{Z}^{d}$, coordinate $\xi_{k}^{x}$ takes values in space $\mathbb{R}^{1}$, called the spin space of $k^{\text {th }}$ particle. 
Process $W(t)=\left\{W_{k}(t)\right\}_{k \in \mathbb{Z}^{d}}, t \geqslant 0$ is formed from independent Wiener processes, running at spin spaces of each particle $k \in \mathbb{Z}^{d}$. Its canonical realizations $\left(\Omega, \mathcal{F}, \mathcal{F}_{t}, \mathcal{W}_{\mathbb{Z}^{d}}\right)$ may be described by fixing some vector $\left\{a_{k}>0\right\}_{k \in \mathbb{Z}^{d}}, \sum_{k \in \mathbb{Z}^{d}} a_{k}=1$, and probability space $\Omega=C_{0}\left(\mathbb{R}_{+}, \ell_{2}(a)\right)$ with Borel $\sigma$-algebra $\mathcal{F}$. The flow of $\sigma$-algebras $\mathcal{F}_{t}$ is formed by events till the time $t$ and $\mathcal{W}_{\mathbb{Z}^{d}}$ is a product of Wiener measures at each point of lattice $k \in \mathbb{Z}^{d}$ [1]. The linear finite-diagonal map $B: \mathbb{R}^{\mathbb{Z}^{d}} \rightarrow \mathbb{R}^{\mathbb{Z}^{d}}$ introduces the interaction between particles of finite radius $r_{0}$. It is defined by a finite set of real numbers $\left\{b_{i}: i \in \mathbb{Z}^{d},|i| \leqslant r_{0}\right\}$ :

$$
(B x)_{k}=\sum_{j \in \mathbb{Z}^{d}:|j-k| \leqslant r_{0}} b_{k-j} x_{j}
$$

and represents a bounded mapping in any space

$$
\ell_{p}(c)=\ell_{p}\left(c, \mathbb{Z}^{d}\right)=\left\{x \in \mathbb{R}^{\mathbb{Z}^{d}}:\|x\|_{\ell_{p}(c)}=\left(\sum_{k \in \mathbb{Z}^{d}} c_{k}\left|x_{k}\right|^{p}\right)^{1 / p}<\infty\right\}
$$

defined by vectors $c=\left\{c_{k}>0\right\}_{k \in \mathbb{Z}^{d}}$, such that $\delta_{c}=\sup _{|k-j|=1}\left|c_{k} / c_{j}\right|<\infty$. Henceforth we denote a set of such vectors $c$ by $\mathbb{P}$.

The mapping $F: \mathbb{R}^{\mathbb{Z}^{d}} \ni\left\{x_{k}\right\}_{k \in \mathbb{Z}^{d}}=x \longrightarrow F(x)=\left\{F\left(x_{k}\right)\right\}_{k \in \mathbb{Z}^{d}} \in \mathbb{R}^{\mathbb{Z}^{d}}$ introduces nonlinearity in the model, i.e gives each particle some potential. It is generated by monotonous increasing $C^{\infty}$-function $F, F(0)=0$, which satisfies the condition of no more than polynomial growth

$$
\exists \mathbf{k} \geqslant-1 \forall i \geqslant 1 \quad\left|F^{(i)}(x)-F^{(i)}(y)\right| \leqslant C_{i}|x-y|(1+|x|+|y|)^{\mathbf{k}} .
$$

We are going to demonstrate that nonlinearity directly effects the regularity properties of $\xi^{x}(t), P_{t}$ and the structure of topologies in the spaces of their regularity.

Let us remark that the above conditions guarantee the solvability of equation (2.1) for the initial data $x \in \ell_{2}(a)$ [1]. Therefore, the associated Feller semigroup is constructed as a mean with respect to the product Wiener measure

$$
\left(P_{t} f\right)(x)=\mathbf{E}\left(f\left(\xi^{x}(t)\right)\right)
$$

Its generator may be calculated on the $C^{\infty}$-function $f$ with compact support, that depend on the finite number of variables $x_{k}$ by formula:

$$
[H f](x)=\sum_{k \in \mathbb{Z}^{d}}\left\{-\frac{1}{2} \partial_{k}^{2}+\left[F\left(x_{k}\right)+\sum_{j \in \mathbb{Z}^{d}} b_{k-j} x_{j}\right] \partial_{k}\right\} f(x),
$$

where we introduced a notation $\partial_{k}=\partial / \partial x_{k}$ for partial derivative. Since each coordinate of solution $\xi^{x}(t)$ fulfills the equation (2.1), the representation of generator (2.4) follows from the finite dimensional Ito formula, applied to the finite number of coordinate processes $\xi_{k}^{x}$.

It is important that operator $H$ also arises as energy operator

$$
(H u, u)_{L_{2}(\mu)}=\frac{1}{2} \int_{\mathbb{R}^{\mathbb{Z}^{d}}} \sum_{k \in \mathbb{Z}^{d}}\left|\partial_{k} u(x)\right|^{2} \mathrm{~d} \mu(x)
$$

of Gibbs lattice measure $\mu$ of the form:

$$
\mathrm{d} \mu(x)=\frac{1}{Z} \exp \left\{-\sum_{i, j \in \mathbb{Z}^{d}:|i-j| \leqslant r_{0}} b_{i-j} x_{i} x_{j}\right\} \prod_{k \in \mathbb{Z}^{d}} \mathrm{e}^{-\Phi\left(x_{k}\right)} \mathrm{d} x_{k}, \quad \Phi\left(x_{k}\right)=2 \int_{0}^{x_{k}} F(z) \mathrm{d} z .
$$

Measure $\mu$ describes the model of anharmonic crystal with a finite radius of interaction $r_{0}$. In particular, measure $\mu$ represents one of the possible lattice approximations to the Euclidean field models with interaction. 
To obtain regularity properties of process $\xi^{x}(t)(2.1)$ and semigroup $P_{t}(2.3)$ let us find the representation for partial derivatives of semigroup $\partial_{\tau} P_{t} f$, where $\tau=\left\{k_{1}, \ldots, k_{n}\right\}$ and $\partial_{\tau}=$ $\partial^{|\tau|} / \partial x_{k_{1}} \ldots \partial x_{k_{n}}$. The formal successive differentiation of $(2.3)$ leads to

$$
\partial_{\tau}\left(P_{t} f\right)(x)=\sum_{s=1}^{m} \sum_{\gamma_{1} \cup \cdots \cup \gamma_{s}=\tau} \mathbf{E}\left\langle\partial^{(s)} f\left(\xi^{x}(t)\right), \xi_{\gamma_{1}}^{x}(t) \otimes \cdots \otimes \xi_{\gamma_{s}}^{x}(t)\right\rangle,
$$

where $\partial^{(s)} f$ denotes a set of all $s^{t h}$ order partial derivatives of function $f: \partial^{(s)} f=\left\{\partial_{\gamma} f\right\}_{|\gamma|=s}$ for $\partial_{\gamma} f=\partial_{j_{1}} \ldots \partial_{j_{s}} f$ with $\gamma=\left\{j_{1}, \ldots j_{s}\right\}$, and we used the notation

$$
\left\langle\partial^{(s)} f\left(\xi^{x}(t)\right), \xi_{\gamma_{1}}^{x} \otimes \cdots \otimes \xi_{\gamma_{s}}^{x}\right\rangle=\sum_{i_{1}, . ., i_{s} \in \mathbb{Z}^{d}}\left(\partial_{\left\{i_{1}, \ldots, i_{s}\right\}} f\right)\left(\xi^{x}(t)\right) \xi_{i_{1}, \gamma_{1}}^{x} \ldots \xi_{i_{s}, \gamma_{s}}^{x}
$$

In (2.6) summation $\sum_{\gamma_{1} \cup \ldots \cup \gamma_{s}=\tau}$ over on all possible subdivisions of the set $\tau=\left\{k_{1}, \ldots, k_{n}\right\}, \quad k_{i} \in$ $\mathbb{Z}^{d}$ over the nonempty nonintersecting subsets $\gamma_{1}, \ldots, \gamma_{s} \subset \tau$, with $\left|\gamma_{1}\right|+\cdots+\left|\gamma_{s}\right|=|\tau|, s \geqslant 2$. In Theorem 4 we will precise a class of functions $f$ for which representation (2.6) becomes rigorous.

Vector $\xi_{\tau}^{x}=\left\{\xi_{i, \tau}^{x}\right\}_{i \in \mathbb{Z}^{d}}$ in (2.6) is derivative of $\xi^{x}(t)$ with respect to the initial data $x=\left\{x_{k}\right\}_{k \in \mathbb{Z}^{d}}$

$$
\xi_{i, \tau}^{x}=\frac{\partial^{|\tau|} \xi_{i}^{x}(t)}{\partial x_{k_{n}} \ldots \partial x_{k_{1}}}
$$

and is called hereinafter a $\tau^{t h}$ variation of $\xi^{x}(t)$.

The equation for $\xi_{\tau}$ is derived by the formal successive differentiation of (2.1) with respect to $x$ :

$$
\left\{\begin{array}{l}
\frac{\mathrm{d} \xi_{i, \tau}^{x}}{\mathrm{~d} t}=-F^{\prime}\left(\xi_{i}^{x}\right) \xi_{i, \tau}^{x}-\sum_{j:|j-i| \leqslant r_{0}} b_{j-i} \xi_{j, \tau}^{x}-\varphi_{i, \tau}^{x} \\
\xi_{k, \tau}(0)=x_{k, \tau}
\end{array}\right.
$$

where $\varphi_{i, \tau}^{x}=\varphi_{i, \tau}^{x}\left(\xi^{x}, \xi_{\cdot, \gamma}^{x}, \gamma \subset \tau, \gamma \neq \tau\right)$.

$$
\varphi_{i, \tau}^{x}=\sum_{\gamma_{1} \cup \ldots \cup \gamma_{s}=\tau, s \geqslant 2} F^{(s)}\left(\xi^{x}\right) \xi_{i, \gamma_{1}} \ldots \xi_{i, \gamma_{s}} .
$$

A precise sense to expression (2.7) as a solution to (2.8) can be given only under the special choice of initial data

$$
\widetilde{x}_{k, \tau}=\left\{\begin{array}{l}
\delta_{k, j}, \quad|\tau|=1, \tau=\{j\} \subset \mathbb{Z}^{d} \\
0, \quad|\tau|>1
\end{array}\right.
$$

Let us turn the attention of the reader to the equation (2.8) which is a linear nonautonomous and inhomogeneous equation with respect to variation $\xi_{\tau}$. Its inhomogeneous part depends on the lower order variations $\xi_{\gamma}, \gamma \subset \tau$ and, displays symmetry (1.2) just like the r.h.s. of (2.6).

Representation (2.6) gives the relation between the partial derivatives of semigroup (2.3) and the behaviour of variations $\xi_{\tau}$ with respect to the initial data $x,\left\{\widetilde{x}_{\gamma}\right\}_{\gamma} \subseteq \tau$. Therefore, to construct a semigroup $P_{t}$ in the spaces of continuously differentiable functions we have to study the variations $\xi_{\tau}^{x}$ of process $\xi^{x}(t)$, i.e. its differentiability with respect to the initial data $x$.

The key idea is that for variational equation (2.8) symmetry (1.2) becomes proportionality: variation $\xi_{\tau}, \tau=\left\{k_{1}, \ldots, k_{n}\right\}$, in the r.h.s. of (2.8) is proportional to the product of first order variations $\prod_{i=1}^{n} \xi_{\left\{k_{i}\right\}}$ in the r.h.s. of (2.8).

Taking into account this observation we introduce a special nonlinear expression

$$
\rho_{\tau}(\xi ; t)=\mathbf{E} \sum_{s=1}^{n}\left\{p_{s}\left(z_{t}\right) \sum_{\gamma \subseteq \tau,|\gamma|=s}\left\|\xi_{\gamma}\right\|_{\ell_{m_{\gamma}}\left(c_{\gamma}\right)}^{m_{\gamma}}\right\}
$$

that reflects this symmetry and, in nonlinear manner, takes into consideration the regularity of the process $\xi^{x}(t)$ with respect to the initial data. Above $\tau=\left\{k_{1}, \ldots, k_{n}\right\}, k_{i} \in \mathbb{Z}^{d}, z_{t}=\left\|\xi^{x}(t)\right\|_{\ell_{2}(a)}^{2}$, 
$m_{\gamma}=m_{1} /|\gamma|,|\gamma|$ is a number of points in set $\gamma \subseteq \mathbb{Z}^{d}$ and each $p_{s}$ is increasing $C^{\infty}$ polynomial that fulfills the condition: $\exists \varepsilon>0, \exists K>0$, such that $\forall z \in \mathbb{R}_{+}$

$$
p_{s}(z) \geqslant \varepsilon \quad \& \quad(1+z)\left(\left|p_{s}^{\prime}(z)\right|+\left|p_{s}^{\prime \prime}(z)\right|\right) \leqslant K p_{s}(z) .
$$

It should be noted that in (2.11) we use notations $\ell_{p}(a)$ and $\ell_{m_{\tau}}\left(c_{\tau}\right)$ for the spaces, where initial process $\xi^{x}(t)$ and variational processes $\xi_{\tau}(t)$ are considered. The main reason is that the process $\xi^{x}(t)$ can be constructed only in space $\ell_{p}(a)$ with $\sum_{k \in \mathbb{Z}^{d}} a_{k}<\infty$. On the other hand, due to initial data (2.10), the choice of weights $c_{\tau} \in \mathbb{P}$ is quite arbitrary.

The following theorem states an a priori estimate on any order regularity of the process $\xi_{t}^{x}$.

Theorem 1 Let $F$ satisfy (2.2), $m_{1}>|\tau|$ be fixed, $m_{\gamma}=m_{1} /|\gamma|$ and $\xi^{x},\left\{\xi_{\tau}\right\}_{\gamma \subseteq \tau}$ be strong solutions to systems (2.1), (2.8). Suppose that functions $p_{s}(z), s=1, \ldots, n$ and vectors $\left\{c_{\gamma}\right\}_{\gamma \subseteq \tau} \subset$ $\mathbb{P}$ in (2.11) fulfill:

(1) $\exists K_{p} \forall j=2, \ldots, n \forall i_{1}, \ldots, i_{s}: i_{1}+\cdots+i_{s}=j, s \geqslant 2$

$$
\left[p_{j}(z)\right]^{j}(1+z)^{\frac{\mathbf{k}+1}{2}} m_{1} \leqslant K_{p} \cdot\left[p_{i_{1}}(z)\right]^{i_{1}} \ldots\left[p_{i_{s}}(z)\right]^{i_{s}} ;
$$

(2) for any subdivision of the set $\gamma=\alpha_{1} \cup \cdots \cup \alpha_{s}, \gamma \subset \tau$ on nonempty nonintersecting subsets $\alpha_{1}, \ldots, \alpha_{s}, s \geqslant 2$ there are constants $R_{\gamma, \alpha_{1}, \ldots, \alpha_{s}}$ such that $\forall k \in \mathbb{Z}^{d}$

$$
\left[c_{k, \gamma}\right]^{|\gamma|} a_{k}^{-\frac{\mathbf{k}_{+1}}{2} m_{1}} \leqslant R_{\gamma, \alpha_{1}, \ldots, \alpha_{s}}\left[c_{k, \alpha_{1}}\right]^{\left|\alpha_{1}\right|} \ldots\left[c_{k, \alpha_{s}}\right]^{\left|\alpha_{s}\right|} .
$$

Upper indexes outside the brackets $[\cdots]$ mean powers and parameter $\mathbf{k}$ is introduced in (2.2).

Then there is a constant $M \in \mathbb{R}^{1}$, such that the nonlinear estimate of exponential type on the a priori regularity of process $\xi_{t}^{x}$ holds

$$
\rho_{\tau}(\xi ; t) \leqslant \mathrm{e}^{M t} \rho_{\tau}(\xi ; 0)
$$

Let us remark that the set of functions $p_{i}$ and vectors $c_{\tau}$, which satisfy the conditions (2.13), (2.14), is sufficiently large. First of all, for $p_{i}$ and $c_{\tau}$ that fulfill (2.13), (2.14) function $q \cdot p_{i}$ and vector $d \cdot c_{\tau}=\left\{d_{k} c_{k, \tau}\right\}_{k \in \mathbb{Z}^{d}}$ again fulfill (2.13) and (2.14), where $d \in \mathbb{P}$ and $q$ fulfills (2.12). An example may be given by

$$
\begin{aligned}
\widetilde{p}_{i} & =q(z)(1+z)^{\frac{\mathbf{k}_{+1}}{2}\left(m_{1} / i-m_{1} /|\tau|\right)}, \\
\widetilde{c}_{k, \gamma} & =a_{k}^{\frac{\mathbf{k}_{+1}}{2} m_{1} \frac{|\gamma|-1}{|\gamma|}} \prod_{j \in \tau} \psi_{k-j}^{m_{1} /|\gamma|}, \quad \gamma=\left\{j_{1}, \ldots, j_{s}\right\}
\end{aligned}
$$

with some polynomial $q$ and vector $\psi=\left\{\psi_{k}\right\}_{k \in \mathbb{Z}^{d}} \in \mathbb{P}$. They fulfill (2.13) and (2.14) with constants $K_{p}=R_{\gamma ; \alpha_{1}, \ldots \alpha_{s}}=1$. Indeed, due to $a_{k} \leqslant 1$

$$
\begin{aligned}
a_{k}^{-\frac{\mathbf{k}_{+1}}{2} m_{1}}\left[\widetilde{c}_{k, \tau}\right]^{|\tau|} & =a_{k}^{\frac{\mathbf{k}_{+1}}{2} m_{1}(|\tau|-2)} \prod_{j \in \tau} \psi_{k-j}^{m_{1}} \leqslant a_{k}^{\frac{\mathbf{k}_{+1}}{2} m_{1}(|\tau|-s)} \prod_{j \in \tau} \psi_{k-j}^{m_{1}} \\
& =\prod_{i=1}^{s}\left[a_{k}^{\frac{\mathbf{k}+1}{2} m_{1} \frac{\left|\gamma_{i}\right|-1}{\left|\gamma_{i}\right|}} \prod_{b \in \gamma_{i}} \psi_{k-b}^{m_{1} /\left|\gamma_{i}\right|}\right]^{\left|\gamma_{i}\right|}=\left[\widetilde{c}_{k, \gamma_{1}}\right]^{\left|\gamma_{1}\right|} \ldots\left[\widetilde{c}_{k, \gamma_{s}}\right]^{\left|\gamma_{s}\right|},
\end{aligned}
$$

where $\tau=\gamma_{1} \cup \cdots \cup \gamma_{s},\left|\gamma_{1}\right|+\cdots+\left|\gamma_{s}\right|=|\tau|, s \geqslant 2$. Similar calculation holds for $\widetilde{p}_{i}$.

Proof. We apply Ito formula to the expression $\rho_{\tau}$ (2.11), then we use symmetries (1.2) and hierarchies (2.13), (2.14) with further application of Gronwall-Bellmann inequality. Introduce notations

$$
g_{\gamma}(t)=\mathbf{E}\left[p_{|\gamma|}\left(z_{t}\right)\left\|\xi_{\gamma}(t)\right\|_{\ell_{m_{\gamma}}\left(c_{\gamma}\right)}^{m_{\gamma}}\right]
$$


where $z_{t}=\left\|\xi^{x}(t)\right\|_{\ell_{2}(a)}^{2}$, and

$$
h_{\tau}^{i}(\xi ; t)=\sum_{s=1}^{i} \sum_{\gamma \subseteq \tau,|\gamma|=s} g_{\gamma}(t)
$$

for $i \geqslant 1, h_{\tau}^{0}(\xi ; t)=0$ for $i=0$. We prove inductively that

$$
\forall i=1, . ., n \quad \exists M_{i} \in \mathbb{R} \quad h_{\tau}^{i}(\xi ; t) \leqslant \mathrm{e}^{M_{i} t} h_{\tau}^{i}(\xi ; 0),
$$

which at $i=n$ gives the statement of theorem. If for any $\gamma \subseteq \tau,|\gamma|=i$ we prove

$$
\frac{\mathrm{d} g_{\gamma}(t)}{\mathrm{d} t} \leqslant K_{1} g_{\gamma}(t)+K_{2} h_{\tau}^{i-1}(\xi ; t)
$$

then Gronwall-Bellmann inequality implies (2.18):

$$
\begin{aligned}
h_{\tau}^{i}(\xi ; t) & \leqslant \mathrm{e}^{M_{i-1} t} h_{\tau}^{i-1}(\xi ; 0)+\sum_{\gamma \subseteq \tau,|\gamma|=i}\left\{\mathrm{e}^{K_{1} t} g_{\gamma}(0)+K_{2} \int_{0}^{t} \mathrm{e}^{K_{1}(t-s)} \mathrm{e}^{M_{i-1} s} h_{\tau}^{i-1}(\xi ; 0) \mathrm{d} s\right\} \\
& \leqslant \mathrm{e}^{\left(M_{i-1}+K_{1}\right) t}\left(1+2^{|\tau|} K_{2} t\right) h_{\tau}^{i}(\xi ; 0) \leqslant \mathrm{e}^{\left(M_{i-1}+K_{1}+2^{|\tau|} K_{2}\right) t} h_{\tau}^{i}(\xi ; 0) .
\end{aligned}
$$

To prove (2.19) let us assume that processes $\xi^{x}(t), \xi_{\gamma}(t), \gamma \subseteq \tau$ are strong solutions to equations (2.1) and (2.8). Therefore $\xi^{x}(t)$ is a sum of Wiener process and finite variation part and $\xi_{\gamma}(t), \gamma \subseteq \tau$ are processes of finite variation. As a consequence, Ito formula can be applied to the expression $g_{\gamma}(t)$, e.g. $[1,3,4,18]$. It gives

$$
\begin{gathered}
p_{i}\left(z_{t}\right)\left\|\xi_{\gamma}(t)\right\|_{\ell_{m_{\gamma}}\left(c_{\gamma}\right)}^{m_{\gamma}}=p_{i}\left(z_{0}\right)\left\|\xi_{\gamma}(0)\right\|_{\ell_{m_{\gamma}}\left(c_{\gamma}\right)}^{m_{\gamma}}+2 \int_{0}^{t} p_{i}^{\prime}\left(z_{s}\right)\left\|\xi_{\gamma}(s)\right\|_{\ell_{m_{\gamma}}\left(c_{\gamma}\right)}^{m_{\gamma}}\left(\xi^{x}(s), \mathrm{d} W(s)\right) \\
+\int_{0}^{t}\left\{m_{\gamma} p_{i}\left(z_{s}\right)\left\langle\frac{\mathrm{d} \xi_{\gamma}(s)}{\mathrm{d} s},\left[\xi_{\gamma}(s)\right]^{\#}\right\rangle_{\ell_{m_{\gamma}\left(c_{\gamma}\right)}}-\left\|\xi_{\gamma}\right\|_{\ell_{m_{\gamma}}\left(c_{\gamma}\right)}^{m_{\gamma}}(H p)\left(z_{s}\right)\right\} \mathrm{d} s .
\end{gathered}
$$

Here

$$
(x, y)=\sum_{k \in \mathbb{Z}^{d}} a_{k} x_{k} y_{k}, \quad\left\langle u, v^{\#}\right\rangle_{\ell_{m}(c)}=\sum_{k \in \mathbb{Z}^{d}} c_{k} u_{k} v_{k} \cdot\left|v_{k}\right|^{m-2}
$$

for $v^{\#}=\|v\|_{\ell_{m}(c)}^{m-2} \mathcal{F} v$ with duality map $\mathcal{F}$ in space $\ell_{m}(c)$ and operator $H$ is defined in (2.4).

Inequality

$$
\left|\left\langle f, \xi^{\#}\right\rangle\right| \leqslant \frac{1}{m}\|f\|_{\ell_{m}(c)}^{m}+\frac{m-1}{m}\|\xi\|_{\ell_{m}(c)}^{m},
$$

property $F^{\prime}(x) \geqslant 0, x \in \mathbb{R}$, the boundedness of map $B$ in any space $\ell_{p}(c), p \geqslant 1, \delta_{c}<\infty$ and inequality $H p_{i}\left(z_{t}\right) \geqslant-M_{p_{i}} p_{i}\left(z_{t}\right)$ (see [10, Hint 9]) imply

$$
\frac{\mathrm{d} g_{\gamma}(t)}{\mathrm{d} t} \leqslant \text { const } g_{\gamma}(t)+\sum_{\alpha_{1} \cup \cdots \cup \alpha_{s}=\gamma, s \geqslant 2} \mathbf{E} p_{i}\left(\left\|\xi^{x}\right\|_{\ell_{2}(a)}^{2}\right)\left\|F^{(s)}\left(\xi^{x}\right) \xi_{\alpha_{1}} \ldots \xi_{\alpha_{s}}\right\|_{\ell_{m_{\gamma}}\left(c_{\gamma}\right)}^{m_{\gamma}} .
$$

Due to (2.2) we have

$$
\left|F^{(s)}\left(\xi_{k}^{x}\right)\right| \leqslant C\left(1+\left|\xi_{k}^{x}\right|\right)^{\mathbf{k}+1} \leqslant C \cdot a_{k}^{-\frac{\mathbf{k}_{+1}}{2}}\left(1+\left\|\xi^{x}(t)\right\|_{\ell_{2}(a)}^{2}\right)^{\frac{\mathbf{k}_{+1}}{2}} .
$$

As $m_{\gamma}=m_{\alpha} \cdot|\alpha| /|\gamma|$ we get

$$
\left|\xi_{k, \alpha_{1}}\right|^{m_{\gamma}} \cdots\left|\xi_{k, \alpha_{s}}\right|^{m_{\gamma}}=\left[\left|\xi_{k, \alpha_{1}}\right|^{m_{\alpha_{1}}}\right]^{\left|\alpha_{1}\right| /|\gamma|} \cdots\left[\left|\xi_{k, \alpha_{s}}\right|^{m_{\alpha_{s}}}\right]^{\left|\alpha_{s}\right| /|\gamma|} .
$$


Therefore, properties (2.13) and (2.14) imply the estimate on each term in (2.21):

$$
(2.21) \leqslant C K_{p} R_{\gamma, \alpha_{1} . . \alpha_{s}} \mathbf{E} \sum_{k \in \mathbb{Z}^{d}} \prod_{i=1}^{s}\left\{p_{\left|\alpha_{i}\right|}\left(|| \xi^{x} \|_{\ell_{2}(a)}^{2}\right) c_{k, \alpha_{i}}\left|\xi_{k, \alpha_{i}}\right|^{m_{\alpha_{i}}}\right\}^{\left|\alpha_{i}\right| /|\gamma|}
$$

Inequality $\left|x_{1} \ldots x_{s}\right| \leqslant \frac{\left|x_{1}\right|^{q_{1}}}{q_{1}}+\cdots+\frac{\left|x_{s}\right|^{q_{s}}}{q_{s}}$ with $q_{j}=|\gamma| /\left|\alpha_{j}\right|$ implies

$$
\begin{aligned}
(2.22) & \leqslant C K_{p} R_{\gamma, \alpha_{1} . . \alpha_{s}} \mathbf{E} \sum_{j=1}^{s} \frac{\left|\alpha_{j}\right|}{|\gamma|} p_{\left|\alpha_{j}\right|}\left(\left\|\xi^{x}\right\|_{\ell_{2}(a)}^{2}\right)\left\|\xi_{\alpha_{j}}\right\|_{\ell_{m_{\alpha_{j}}}\left(c_{\alpha_{j}}\right)}^{m_{\alpha_{j}}} \\
& \leqslant C K_{p} R_{\gamma, \alpha_{1}, . ., \alpha_{s}} h_{\tau}^{i-1}(\xi ; t)
\end{aligned}
$$

In the last inequality we assumed that for subdivision $\alpha_{1} \cup \cdots \cup \alpha_{s}=\gamma,|\gamma|=i$ at $s \geqslant 2$ we have $\left|\alpha_{j}\right| \leqslant i-1$. Therefore (2.19) is proved.

\section{3. $C^{\infty}$-regularity of semigroup $P_{t}$}

Now we can discuss the structure of topologies in spaces, in which the differentiability properties of semigroup $P_{t}$ hold. It is determined by nonlinear estimate (2.15) and essentially depends on the order of nonlinearity $\mathbf{k}$ of map $F$, which is reflected in special hierarchy of weights in seminorms. In [10] it is demonstrated that such hierarchy of weights is non-void, if the semigroup is constructed in the spaces of differentiable functions.

Introduce Banach space $\operatorname{Lip}_{r}\left(\ell_{2}(a)\right), r \geqslant 0$, equipped with norm

$$
\|f\|_{L_{i p_{r}}}=\sup _{x \in \ell_{2}(a)} \frac{|f(x)|}{\left(1+\|x\|_{\ell_{2}(a)}\right)^{r+1}}+\sup _{x, y \in \ell_{2}(a)} \frac{|f(x)-f(y)|}{\|x-y\|_{\ell_{2}(a)}\left(1+\|x\|_{\ell_{2}(a)}+\|y\|_{\ell_{2}(a)}\right)^{r}}<\infty .
$$

For $m \in \mathbb{N}$, we denote a finite array of weights $\left\{(q, \mathcal{G}):(q, \mathcal{G}) \in \Theta^{m}\right\}$ by $\Theta^{m}$, where $\mathcal{G}=$ $G^{1} \otimes \cdots \otimes G^{m}$ is $m$-tensor constructed from vectors $G^{i} \in \mathbb{P}, i=1, \ldots, m$ and $q$ is a smooth function that fulfills (2.12).

Definition 2 Let $r \geqslant 0, n \geqslant 1$ and $\Theta=\Theta^{1} \cup \cdots \cup \Theta^{n}, \Theta^{i} \neq \emptyset, i=1, \ldots, n$ be a finite array of weights. Function $f$ belongs to the space of continuously differentiable functions $C_{\Theta, r}\left(\ell_{2}(a)\right)$ iff $f \in \operatorname{Lip}_{r}\left(\ell_{2}(a)\right)$ and

1) for any $m \in\{1, \ldots, n\}$ and $\tau=\left\{j_{1}, \ldots, j_{m}\right\}, j_{i} \in \mathbb{Z}^{d}$, there is a continuous partial derivative $\partial_{\tau} f \in C\left(\ell_{2}(a), \mathbb{R}^{1}\right)$. These derivatives fulfill integral relations: $\forall x \in \ell_{2}(a), \forall h \in \mathbf{X}_{\infty}([a, b])$

$$
\left.f(x+h(\cdot))\right|_{a} ^{b}=\int_{a}^{b} \mathrm{~d} s \sum_{k \in \mathbb{Z}^{d}} \partial_{k} f(x+h(s)) h_{k}^{\prime}(s)
$$

and $\forall \tau=\left\{j_{1}, \ldots, j_{\ell}\right\},|\tau|=\ell \leqslant n-1$

$$
\left.\partial_{\tau} f(x+h(\cdot))\right|_{a} ^{b}=\int_{a}^{b} \mathrm{~d} s \sum_{k \in \mathbb{Z}^{d}} \partial_{\tau \cup\{k\}} f(x+h(s)) h_{k}^{\prime}(s) .
$$

Here we used the notation

$$
\mathbf{X}_{\infty}([a, b])=\bigcap_{p \geqslant 1, c \in \mathbb{P}} A C_{\infty}\left([a, b], \ell_{p}(c)\right)
$$

where $A C_{\infty}([a, b], X)=\left\{h \in C([a, b], X): \exists h^{\prime} \in L_{\infty}([a, b], X\}\right.$ for Banach space $X$. 
2) The norm is finite

$$
\|f\|_{C_{\Theta, r}}=\|f\|_{L i p_{r}}+\max _{m=1, n}\left\|\partial^{(m)} f\right\|_{\Theta^{m}}<\infty
$$

where

$$
\left\|\partial^{(m)} f\right\|_{\Theta^{m}}=\sup _{x \in \ell_{2}(a)} \max _{\left(q_{m}, \mathcal{G}^{m}\right) \in \Theta^{m}} \frac{\left|\partial^{(m)} f(x)\right|_{\mathcal{G}^{m}}}{q_{m}\left(\|x\|_{\ell_{2}(a)}^{2}\right)}
$$

$$
\text { with }\left|\partial^{(m)} f(x)\right|_{\mathcal{G}^{m}}^{2}=\sum_{\tau=\left\{j_{1} \ldots j_{m}\right\} \subset \mathbb{Z}^{d}} G_{j_{1}}^{1} \ldots G_{j_{m}}^{m}\left|\partial_{\tau} f(x)\right|^{2} \text { for } \mathcal{G}^{m}=G^{1} \otimes \cdots \otimes G^{m} \text {. }
$$

Remark. Definition of $C_{\Theta, r}$ is not transparent at the first glance and we would like to give some comments.

For fixed $\omega \in \Omega, t \in[0 . T]$ the $\operatorname{map} \ell_{2}(a) \ni x \rightarrow \xi^{x}(\omega, t) \in \ell_{2}(a)$ and its variations $\left\{\xi_{\tau}\right\}$ have nonlinear responses with respect to initial data in representation of $\partial_{\tau} P_{t} f(2.6)$. This circumstance motivated us to give a sense $(3.2),(3.3)$ to the derivatives of function $f \in C_{\Theta, r}$. It may be considered as the existence of Frechet derivatives on some projective limit of spaces.

In particular, properties (3.5) and (3.2), (3.3) establish that for function $f \in C_{\Theta, r}$ there exist continuous partial derivatives up to the order $n$. To show this one should take $h(t)=t e^{k}, t \in[0,1]$ with vector $e^{k}=\left\{\delta_{j}^{k}\right\}_{j \in \mathbb{Z}^{d}}$ in (3.2) and (3.3). Due to the finiteness of norm $\|f\|_{C_{\Theta, r}}$ the r.h.s. of (3.2) and (3.3) are well-defined for such $h$.

In the next definition we introduce a special hierarchy of weights in topology of space $C_{\Theta, r}$ that guarantees the regularity properties of semigroup $P_{t}$.

Definition 3 Finite array $\Theta=\Theta^{1} \cup \cdots \cup \Theta^{n}, n \in \mathbb{N}$ is subordinated to the nonlinearity of order $\mathbf{k}$ iff $\forall m=2, \ldots, n$, for any $\operatorname{pair}\left(q, \mathcal{G}=G^{1} \otimes \cdots \otimes G^{m}\right) \in \Theta^{m}$ and $\forall i, j \in\{1, \ldots, m\}, i \neq j$, there is a pair $\left(\widetilde{q}, \widetilde{\mathcal{G}}=\widetilde{G}^{1} \otimes \cdots \otimes \widetilde{G}^{m-1}\right) \in \Theta^{m-1}$ such that

$$
\exists K: \quad \forall z \in \mathbb{R}_{+}^{1} \quad(1+z)^{\frac{\mathbf{k}_{+1}}{2}} \widetilde{q}(z) \leqslant K q(z) ; \forall \ell=1, \ldots, m-1 \quad\left(\widehat{\mathcal{G}}^{\{i, j\}}\right)^{\ell} \leqslant K \widetilde{G}^{\ell} .
$$

In (3.7) $(m-1)$-tensor $\widehat{\mathcal{G}}^{\{i, j\}}$ is constructed from $m$-tensor $\mathcal{G}$ by rule

$$
\widehat{\mathcal{G}}^{\{i, j\}}=G^{1} \otimes \underset{\hat{i}}{\ldots} \underset{\uparrow j}{\ldots} \otimes A^{-(\mathbf{k}+1)} G^{i} G^{j} \otimes . . \otimes G^{m},
$$

with vector $A^{-(\mathbf{k}+1)}=\left\{a_{k}^{-\mathbf{k}+1}\right\}_{k \in \mathbb{Z}^{d}}$. Notation $G^{1} \otimes \ldots \underset{i}{\stackrel{i}{i}} \otimes G^{s}$ means that in tensor product, the $i^{\text {th }}$ - vector is omitted and $G^{1} \otimes . . \otimes \underset{\uparrow j}{B} \otimes . . \otimes G^{s}$ means that on the $j^{\text {th }}$ place in tensor product, $\uparrow j$

there is an inserted vector B. For each $\ell$, inequality (3.7) is understood as a coordinate inequality between two vectors (i.e. $c=\left\{c_{k}\right\} \leqslant d=\left\{d_{k}\right\}$ iff $\forall k c_{k} \leqslant d_{k}$ ).

Let us remark that the structure of seminorm $\|\cdot\|_{\Theta^{m}}$ and condition (3.7) on $\Theta$ is dictated by nonlinear estimate and guarantees that the exponential estimate on semigroup $P_{t}$ is scale $C_{\Theta, r}$. In particular, in [10] it was demonstrated that the hierarchies (3.7) are unavoidable if one wishes to have a property $(3.8)$ for nonlinear $F$.

The next Theorem states the continuous differentiability of semigroup $P_{t}$ on the functions from scale $C_{\Theta, r}$. Let us note that this result holds for multiparticle interactions in Gibbs measure and this result will appear elsewhere.

Theorem 4 Let $F$ fulfill (2.2) with parameter $\mathbf{k}, r \geqslant 0$ and finite array $\Theta=\Theta^{1} \cup \cdots \cup \Theta^{n}$ be subordinated to the nonlinearity of order $\mathbf{k}$. Then $\forall t \geqslant 0: P_{t}: C_{\Theta, r} \rightarrow C_{\Theta, r}$ and $\exists K_{\Theta, r}, M_{\Theta, r}$

$$
\forall f \in C_{\Theta, r} \quad\left\|P_{t} f\right\|_{C_{\Theta, r}} \leqslant K_{\Theta, r} \mathrm{e}^{M_{\Theta, r} t}\|f\|_{C_{\Theta, r}} .
$$

In particular, $\forall f \in C_{\Theta, r}$ derivatives of semigroup fulfill representation (2.6).

Proof. A detailed proof will appear in [11, Ch.4]. 


\section{Acknowledgements}

Authors are grateful to the referee for careful reading the manuscript as well as for making several suggestions for its improvement. A warm hospitality of organizers during the conference in Lviv is much appreciated.

\section{References}

1. Da Prato G., Zabczyk J., Stochastic equations in infinite dimensions. Encyclopedia of Math. and its Appl., 1992, 44.

2. Cerrai S., Second order PDE's in finite and infinite dimension. A probabilistic approach. Lecture Notes in Mathematics, 2001, 1762.

3. Krylov N.V., Rozovskii B.L. On the evolutionary stochastic equations. Contemporary Problems of Mathematics, 14, p. 71-146. VINITI, Moscow, 1979.

4. Pardoux E., Stochastic partial differential equations and filtering of diffusion processes. Stochastics, 1979, 3, 127-167.

5. Glimm J., Jaffe A. Quantum Physics. A Functional Integral Point of View. Springer Verlag, New-York, 1987.

6. Simon B. The $P(\phi)_{2}$ Euclidean (Quantum) Field Theory. Princeton Univ. Press, Princeton, 1974.

7. Daletskii Yu.L., Fomin S.V. Measures and Differential Equations on Infinite Dimensional Spaces. Nauka, Mockow, 1984; Kluwer Academic Publishers, 1992.

8. Bogachev V.I., Da Prato G., Roeckner M., Sobol Z., Gradient bounds for solutions of elliptic and parabolic equations. http://arxiv.org/math.pr/0507079.

9. Athreya S.R., Bass R.F., Gordina M., Perkins E.A., Infinite dimensional stochastic differential equations of Ornstein-Uhlenbeck type. http://arxiv.org/math.pr/0503165.

10. Antoniouk A.Val., Antoniouk A.Vict., How the unbounded drift shapes the Dirichlet semigroups behaviour of non-Gaussian Gibbs measures. Journ. Funct. Anal., 1996, 135, 488-518.

11. Antoniouk A.Val., Antoniouk A.Vict. Nonlinear Effects in the Regularity Properties of the Infinite Dimensional Evolutions of the Classical Gibbs Models. Nauk. Dumka, Kiev, to appear in 2006, 208 p. (in Russian).

12. Bismut J.-M. Large deviations and the Malliavin calculus. Progress in Mathematics, vol. 45. Birkhäuser, 1984.

13. Malliavin P. Stochastic Analysis. Springer Verlag, 1997.

14. Antoniouk A.Val., Antoniouk A.Vict. Non-Lipschitz singularities in the Malliavin calculus: Raise of Smoothness for infinite dimensional semigroups, No. 96.23, 40 p. Inst. Math. Publishing, Kiev, 1996.

15. Antoniouk A.Val., Antoniouk A.Vict., High order formulae for derivatives of nonlinear diffusion semigroups. Ukrainian Math. Journal, January 2001, No. 1, 117-122.

16. Antoniouk A.Val., Antoniouk A.Vict. Nonlinear estimates approach to the regularity properties of diffusion semigroups. Nonlinear Analysis and Applications: To V.Lakshmikantham on his 80th birthday, eds. Ravi P. Agarwal and Donal O'Regan, vol. 1, 165-226 p. Kluwer, 2003.

17. Antoniouk A.Val., Antoniouk A.Vict., Nonlinear Calculus of variations for differential flows on manifolds: geomentrically correct generalization of covariant and stochastic variations. Ukrainian Math. Bulletin, 2004, 4, 449-484.

18. De Prato G., Zabczyk J., Evolution equations with white-noise boundary conditions. Stochastics and Stochastic Reports, 1993, 42, 167-182. 


\section{Нелінійні ефекти в задачах регулярності для нескінченновимірних еволюцій необмежених спінових систем}

Олександр Валерійович Антонюк, Олександра Вікторівна Антонюк

Інститут математики НАН України,

вул. Терещенківська 3, 01601 Київ, Україна

Отримано 15 серпня 2005 р., в остаточному вигляді -

31 січня $2006 \mathrm{p}$.

Досліджено задачі регулярності для необмежених спінових систем ангармонійних осциляторів, що апроксимують євклідові теорії поля у високих розмірностях. Основну увагу приділено впливу ангармонізму на $C^{\infty}$-регулярні властивості еволюційної напівгрупи. Підхід спирається на новий клас нелінійних оцінок на варіації, що дозволяють досліджувати регулярні властивості для істотно нелінійних рівнянь.

Ключові слова: ангармонійні граткові спінові системи, нелінійні задачі регулярності

PACS: 02.30.Jr, 02.30.Sa, 02.30.Xx, 02.50.Eu,03.654.Db, 05.50.+q, 75.10.Hk 